ENZYMES

\section{A Crying Inhibitor}

from our Cell Biology Correspondent

THERE is clearly more to tears than meets the eye, for, as Kueppers has just reported (Biochim. Biophys. Acta, 229. 845 ; 1971), human tears contain an effective inhibitor of the proteolytic enzymes trypsin and chymotrypsin. Motivated by the discovery of inhibitors of trypsin in human urine and pancreatic juice and at least five distinct substances in human serum which inhibit trypsin, chymotrypsin and elastase, Kueppers apparently cast around for another body fluid to test, and hit upon tears. The snag that human tears are, in the adult world, obtainable in only small amounts was overcome with liberal whiffs of ammonia and a sensitivity assay for proteolytic activity, namely the spectroscopic measurement of nitroaniline liberated by trypsin from $\mathbf{N} \alpha$-benzoylDL-arginine- $p$-nitroanilide- $\mathrm{HCl}$.

After giving his subjects whiffs of ammonia, Kueppers took 0.02-0.05 ml. of tears from each weeping eye and tested it against $1 \mu \mathrm{g}$ of trypsin in $1 \mathrm{ml}$. of a 1 per cent aqueous solution of substrate. $1 \mathrm{ml}$. of these induced tears inhibited between $10 \mu \mathrm{g}$ and $35 \mu \mathrm{g}$ of trypsin and the unstimulated lachrymal fluid collected from two subjects had enough inhibitor to block $43 \mu \mathrm{g}$ and $25 \mu \mathrm{g}$ of trypsin.

Using thin layer chromatography, Kueppers was able to show that the inhibitor in tears differs from the previously characterized protease inhibitors found in various other human body fluids. But with a weight in the range 3,000 to 9,000 daltons, the chromatographic and electrophoretic mobilities of the human tear inhibitor recall those of an inhibitor of proteases found in bovine body fluids. The human factor is, however, more susceptible to heat inactivation than its bovine counterpart. As to the physiological role of this substance, it can only be surmised that it may be in tears to protect the cornea and conjunctiva.

\section{IONOPHORES}

\section{Peptide Isolated}

from our Membrane Correspondent AN ionophore is a compound which is capable of reacting with a simple ion to give a complex which shows a preferential partition from water into a hydrophobic phase. Ionophores thus effect the translocation of the complexed ions across natural and artificial lipid membranes. Most of the ionophores so far described are produced by microorganisms or fungi and until recently none had been demonstrated as endogenous products in higher organisms.

Ionophores are given an important role in D. E. Green's model of active transport across the mitochondrial membrane, and last year Blondin and Green postulated that ionophores were endogenous to mitochondria of beef heart. Blondin, DeCastro and Senior (Biochem. Biophys. Res. Commun., 43, 28 ; 1971) have now reported the isolation and partial characterization of just such an ionophore. Because the ionophore valinomycin produces effects in mitochondria similar to the postulated properties of the endogenous ionophore, Blondin et al. were able to work out a successful chromatographic procedure for isolating the endogenous ionophore by assuming that its chromatographic behaviour would be similar to that of valinomycin. By again using valinomycin as a model they were able to assay the endogenous ionophore by its stimulation of potassium ion accumulation from solutions of low potassium acetate concentration into energized mitochondria. Because of its very low concentration in mitochondria, they were unable to isolate enough material to completely characterize the new ionophore, but their results indicate that it is a cyclic peptide with an approximate molecular weight of 1100 which complexes sodium and potassium to about the same degree and they tentatively suggest the structure (Pro. Leu. Val. Ser.) ${ }_{3}$.

This successful isolation of an endogenous ionophore from mitochondria should stimulate a search for ionophores in other mammalian cell membranes. Perhaps the discovery of a calcium-specific ionophore in the sarcoplasmic reticulum can be anticipated, and Hyman (Fed. Proc., 30, 314 ; 1971) has presented some rather inconclusive evidence which suggests that an ionophore may exist in erythrocyte membranes. There are grounds for thinking, however, that the mitochondrial membrane has a unique structure from which generalizations regarding other membranes are largely guesswork. If this view is right, the ionophore search party is likely to return empty handed.

\section{ICE CAPS \\ Selenium Pollution?}

from our Geomagnetism Correspondent

From the purely scientific point of view the relative permanence of the major ice caps is one of their most endearing properties. For much of the ice which

\title{
Strong Pulses and the Crab Pulsar
}

IT was, of course, the occasional unusually strong radio pulses emitted by one of the stars in the Crab Nebula that identified it as a pulsar even before its periodicity was recognized. A special study of the strong pulses is reported in next Monday's Nature Physical Science by Carl Heiles and John M. Rankin, based on observations at Arecibo Observatory, Puerto Rico. Their work refers to low radio frequencies where the contribution of the nebula tends to swamp all but the stronger pulsar pulses in any case. Although Heiles and Rankin do not speculate on the origin of the pulses, their work shows that the strong pulses have other unusual features which will require a special explanation.

The work reported by Heiles and Rankin was done basically in two stages. In one experiment the strong pulses were recorded simultaneously in two channels at $318 \mathrm{MHz}$ and 111 $\mathrm{MHz}$, and in the second experiment at $111 \mathrm{MHz}$ and $74 \mathrm{MHz}$. Data obtained at $430 \mathrm{MHz}$ by the same observers were also available.

On the face of it, the most striking feature of the observations is that strong pulses at $318 \mathrm{MHz}$ do not usually correspond to particularly strong pulses at $111 \mathrm{MHz}$, and vice versa. In other words, the spectral index varies quite unusually. This immediately contrasts with all other pulsars where there is no case when pulse energies at two frequencies are not correlated. Comparison between pulses at $111 \mathrm{MHz}$ and 74 $\mathrm{MHz}$ could not be expressed so definitely by Heiles and Rankin because only six strong pulses were detected at the lower frequency during the observations. In general, however, they remark that at each frequency it is possible to detect between twenty and forty strong pulses in $2 \frac{1}{2}$ hours of observation. In other words, they are writing about a type of pulse which occurs about once every five minutes on average. And they point out that interstellar scintillations probably have a negligible effect on the results.

So Heiles and Rankin use their data to produce some expressions for the frequency dependence of the strong pulses. It is this part of their article which will be quoted in the future theoretical work which one day will account for the occurrence of the strong pulses. At present, however, the immediate problem is to explain the evidence for the variability of the spectral index of the strong pulses. Values between zero and three, and possibly even greater variations, can occur. 